\title{
Chloroquine does not inhibit infection of human lung cells with SARS-CoV-2
}

https://doi.org/10.1038/s41586-020-2575-3

Received: 8 May 2020

Accepted: 16 July 2020

Published online: 22 July 2020

Check for updates

\author{
Markus Hoffmann ${ }^{1,2 \bowtie}$, Kirstin Mösbauer ${ }^{3,4}$, Heike Hofmann-Winkler', Artur Kaul', \\ Hannah Kleine-Weber, ${ }^{1,2}$, Nadine Krüger', Nils C. Gassen ${ }^{5}$, Marcel A. Müller ${ }^{3,4,6}$, \\ Christian Drosten ${ }^{3,4}$ \& Stefan Pöhlmann ${ }^{1,2 凶}$
}

\begin{abstract}
The coronavirus disease 2019 (COVID-19) pandemic, which is caused by severe acute respiratory syndrome coronavirus 2 (SARS-CoV-2), has been associated with more than 780,000 deaths worldwide (as of 20 August 2020). To develop antiviral interventions quickly, drugs used for the treatment of unrelated diseases are currently being repurposed to treat COVID-19. Chloroquine is an anti-malaria drug that is used for the treatment of COVID-19 as it inhibits the spread of SARS-CoV-2 in the African green monkey kidney-derived cell line $\mathrm{Vero}^{1-3}$. Here we show that engineered expression of TMPRSS2, a cellular protease that activates SARS-CoV-2 for entry into lung cells ${ }^{4}$, renders SARS-CoV-2 infection of Vero cells insensitive to chloroquine. Moreover, we report that chloroquine does not block infection with SARS-CoV-2 in the TMPRSS2-expressing human lung cell line Calu-3. These results indicate that chloroquine targets a pathway for viral activation that is not active in lung cells and is unlikely to protect against the spread of SARS-CoV- 2 in and between patients.
\end{abstract}

Chloroquine and hydroxychloroquine are used for the treatment of malaria and have been widely used to treat patients with COVID-19. Both of these drugs are currently under investigation in more than 80 registered clinical trials for the treatment of COVID-19 worldwide ${ }^{2,3}$. Chloroquine and hydroxychloroquine inhibit the ability of SARS-CoV-2 to infect Vero cells $s^{1,5,6}$, providing a rational for using these drugs for the treatment of COVID-19. However, it is unknown whether these drugs inhibit the infection of lung cells and it is poorly understood how they inhibit infection with SARS-CoV-2.

Chloroquine and hydroxychloroquine increase the endosomal $\mathrm{pH}$ of cells and inhibit viruses that depend on low $\mathrm{pH}$ for cell entry ${ }^{7}$. We investigated whether these drugs could also block the cell entry by SARS-CoV-2 and whether entry inhibition accounted for the prevention of infection with SARS-CoV-2. Moreover, we investigated whether entry inhibition is cell-type-dependent, as the virus can use $\mathrm{pH}$-dependent and $\mathrm{pH}$-independent pathways for entry into cells. The spike (S) protein of SARS-CoV-2, which mediates viral entry, is activated by the endosomal-pH-dependent cysteine protease cathepsin $\mathrm{L}$ (CTSL) in some cell lines ${ }^{4}$. By contrast, entry into airway epithelial cells, which express low levels of $\mathrm{CTSL}^{8}$, depends on the $\mathrm{pH}$-independent, plasma-membrane-resident serine protease TMPRSS2 ${ }^{4}$. Notably, the use of CTSL by coronaviruses is restricted to cell lines ${ }^{8-10}$, whereas TMPRSS2 activity is essential for the spread and pathogenesis of the virus in the infected host $\mathrm{t}^{11,12}$.

We compared the inhibition by chloroquine and hydroxychloroquine of S-mediated entry into Vero (kidney), TMPRSS2-expressing Vero and Calu-3 (lung) cells. Calu- 3 cells, as with the airway epithelium, express low amounts of $\mathrm{CTSL}^{8}$ and SARS-CoV-2 entry into these cells is dependent on TMPRSS2 ${ }^{4}$. By contrast, entry of SARS-CoV-2 into Vero cells is CTSL-dependent, and both CTSL and TMPRSS2 support entry into TMPRSS2-expressing Vero cells ${ }^{4}$. As a control, we used camostat mesylate, which inhibits TMPRSS2-dependent entry into cells ${ }^{4}$.

Treatment with camostat mesylate did not interfere with cell viability, whereas chloroquine and hydroxychloroquine slightly reduced the viability of Vero, TMPRSS2-expressing Vero and Calu-3 cells when applied at the highest concentration (Fig. 1a). Inhibition of S-driven entry by camostat mesylate was observed only in TMPRSS2 ${ }^{+}$cell lines, as expected (Fig. 1a and Table1). Moreover, chloroquine and hydroxychloroquine inhibited S-driven entry into TMPRSS2 ${ }^{-}$Vero cells with high efficiency whereas the inhibition of entry into TMPRSS2 ${ }^{+}$Calu- 3 and TMPRSS2 ${ }^{+}$Vero cells was inefficient and absent, respectively (Fig. 1a and Table 1). Therefore, chloroquine and hydroxychloroquine can block S-driven entry, but this inhibition is cell-line-dependent and efficient inhibition is not observed in TMPRSS2 $2^{+}$lung cells.

We next investigated whether the cell-type-dependent differences in entry inhibition translated into differential inhibition of authentic SARS-CoV-2. Indeed, chloroquine efficiently blocked SARS-CoV-2 infection of Vero kidney cells, as expected ${ }^{1}$, but did not efficiently inhibit SARS-CoV-2 infection of Calu-3 lung cells (Fig. 1b, c). A subtle reduction in SARS-CoV-2 infection was seen in the presence of $100 \mu \mathrm{M}$ chloroquine, consistent with the modest inhibition of cellular entry of S-bearing pseudotypes under those conditions (Fig. 1a), but this effect was not statistically significant. In summary, chloroquine did not efficiently block the infection of Calu-3 cells with S-bearing pseudotypes and authentic SARS-CoV-2, indicating that-in these cells-chloroquine does not appreciably interfere with viral entry or the subsequent steps of the viral replication cycle.

${ }^{1}$ Infection Biology Unit, German Primate Center - Leibniz Institute for Primate Research, Göttingen, Germany. ${ }^{2}$ Faculty of Biology and Psychology, Georg-August-University Göttingen, Göttingen, Germany. ${ }^{3}$ Institute of Virology, Charité-Universitätsmedizin Berlin, corporate member of Freie Universität Berlin, Humboldt-Universität zu Berlin, Berlin Institute of Health, Berlin,

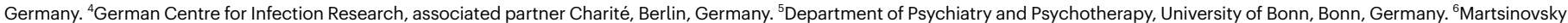
Institute of Medical Parasitology, Tropical and Vector Borne Diseases, Sechenov University, Moscow, Russia. ${ }^{凶}$ e-mail: mhoffmann@dpz.eu; spoehlmann@dpz.eu 

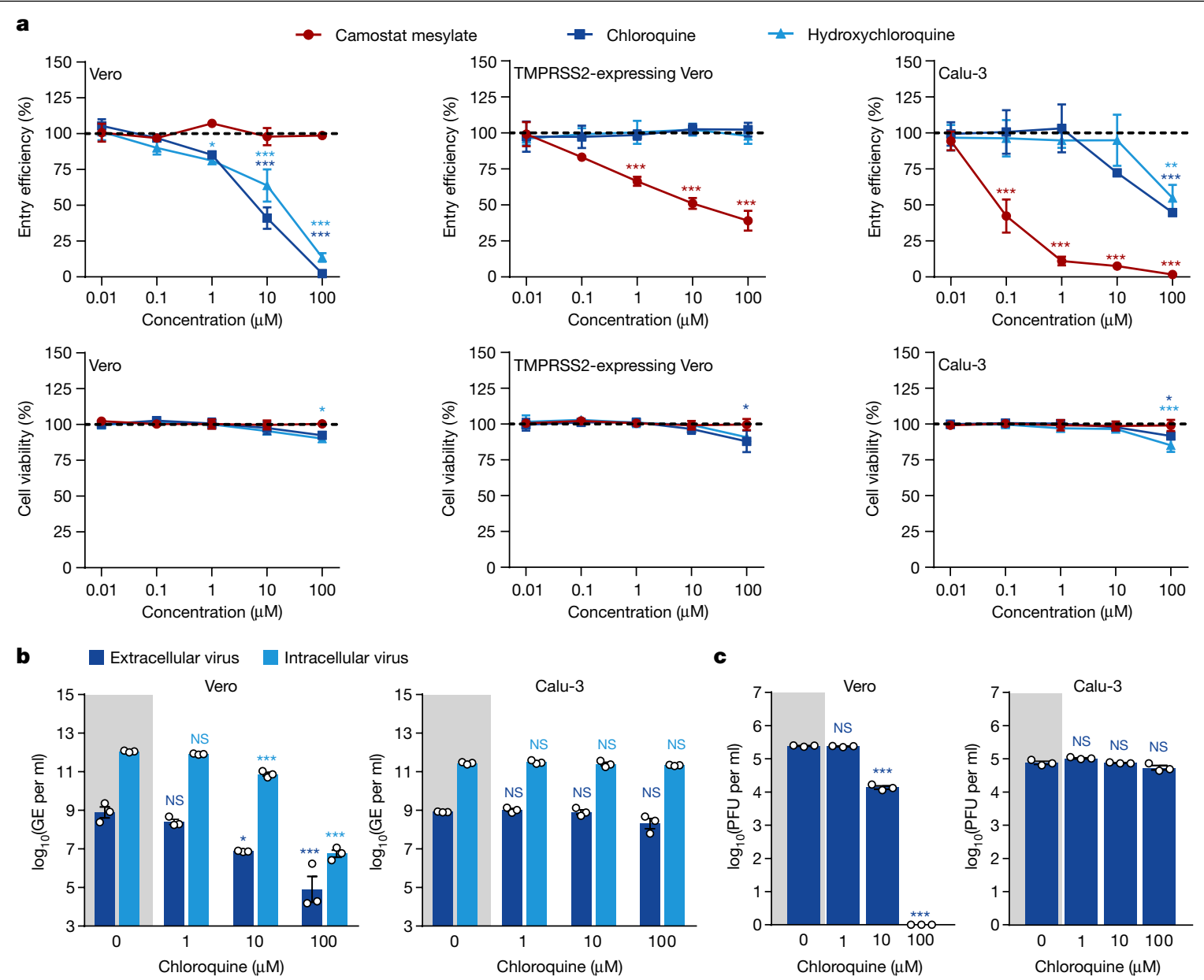

Fig. 1 | Chloroquine does not block infection of human lung cells with SARS-CoV-2. a, Vero, TMPRSS2-expressing Vero and Calu-3 cells were preincubated for $2 \mathrm{~h}$ with the respective inhibitors $(0 \mu \mathrm{M}, 0.01 \mu \mathrm{M}, 0.1 \mu \mathrm{M}$, $1 \mu \mathrm{M}, 10 \mu \mathrm{M}$ or $100 \mu \mathrm{M})$ and then inoculated with replication-defective vesicular stomatitis virus reporter particles bearing the S protein. Top, the transduction efficiency of the virus was assessed. Bottom, cells were not inoculated with virus particles but cell viability after drug treatment was instead assessed at the same time as transduction was quantified. Transduction efficiency was quantified by measuring virus-encoded luciferase activity in cell lysates. Cell viability was measured using the CellTiter-Glo assay. Data are mean \pm s.e.m. of three biological replicates, each of which consisted of quadruplicate samples. Data were normalized as the relative entry efficiency or cell viability of inhibitor-treated cells compared with those of untreated cells (set to $100 \%$ ). The calculated $50 \%$ inhibitory concentration $\left(\mathrm{IC}_{50}\right)$ values are summarized in Table 1. b, Untreated or chloroquine-preincubated Vero and Calu-3 cells were inoculated with SARS-CoV-2 Munich isolate (patient isolate 929, BetaCoV/Munich/BavPat1/2020|EPI_ISL_406862) at a multiplicity of infection (MOI) of 0.001. After inoculation for $24 \mathrm{~h}$, viral RNA was isolated from the culture supernatant (extracellular virus) (dark blue) and the infected cells (intracellular virus) (light blue), and SARS-CoV-2 genome equivalents (GE) were determined by quantitative $P C R$ with reverse transcription. Data are mean \pm s.e.m. of three biological replicates, each of which consisted of single

Research to confirm our results in primary respiratory epithelium is ongoing. Moreover, virus production in Calu-3 cells relative to Vero E6 cells was more robust in the present study compared with a previously published study ${ }^{13}$, potentially due to the use of the Calu-3 subclone 2B4 in the previous but not the present study. Nevertheless, our results suggest that chloroquine and hydroxychloroquine will exert no antiviral activity in human lung tissue and will not be effective against COVID-19, in keeping with the results of recent clinical trials ${ }^{14,15}$. Moreover, our results highlight the fact that cell lines that mimic important aspects of samples. $\mathbf{c}$, The experiment was conducted as described in $\mathbf{b}$, but the number of infectious SARS-CoV-2 particles in culture supernatants was determined by plaque titration using Vero E6 cells. PFU, plaque-forming units. Statistical significance was analysed by two-way analysis of variance (ANOVA) with Dunnett's post hoc test. NS, not significant $(P>0.05)$; ${ }^{*} P \leq 0.05$; ${ }^{* *} P \leq 0.01$; ${ }^{* * *} P \leq 0.001$. $P$ values (from left to right) are as follows. a, Entry efficiency (camostat mesylate/chloroquine/hydroxychloroquine), Vero (0.9999/0.8587/0.9997, 0.9842/0.9846/0.3904, 0.6860/0.0991/0.0223, 0.9968/0.0001/0.0001, 0.9997/0.0001/0.0001), TMPRSS2-expressing Vero $(0.9999 / 0.9968 / 0.9795,0.1251 / 0.9962 / 0.9998,0.0004 / 0.9997 /$ 0.9999, 0.0001/0.9967/0.9982, 0.0001/0.9981/0.9986; Calu-3 (0.9900/ 0.9999/0.9986, 0.0003/0.9999/0.9983, 0.0001/0.9988/0.9929, 0.0001/ $0.1291 / 0.9938,0.0001 / 0.0005 / 0.0045$ ); cell viability (camostat mesylate/ chloroquine/hydroxychloroquine), Vero (0.9273/0.9999/0.9999, 0.9999/ 0.8710/0.9642, 0.9999/0.9996/0.9999, 0.9999/0.8958/0.4818, 0.9998/ 0.0838/0.0161), TMPRSS2-expressing Vero $(0.9998 / 0.9999 / 0.9959$, 0.9811/0.9985/0.9362,0.9998/0.9985/0.9997, 0.9997/0.8835/0.9998, 0.9999/0.0315/0.1422), Calu-3 (0.9986/0.9999/0.9999, 0.9999/0.9997/0.9999, 0.9986/0.9999/0.8134, 0.9924/0.9275/0.7125,0.9983/0.0492/0.0002). b, (extracellular/intracellular), Vero $(0.6844 / 0.6989,0.0121 / 0.0002,0.0002 /$ 0.0001), Calu-3 (0.9434/0.8800, 0.9999/0.8830, 0.0517/0.3924).c, (extracellular/ intracellular), Vero (0.9561, 0.0001, 0.0001), Calu-3 (0.1184, 0.9997, 0.0987).

Table 1 | Half-maximal inhibitory concentrations of the tested drugs

\begin{tabular}{lccc}
\hline & \multicolumn{3}{c}{ IC $_{50}(\boldsymbol{\mu M})$} \\
\cline { 2 - 4 } & Vero & TMPRSS2 $^{+}$Vero & Calu-3 \\
\hline Camostat mesylate & ND & 5.7 & 0.083 \\
\hline Chloroquine & 6.5 & ND & 64.7 \\
\hline Hydroxychloroquine & 13.3 & ND & 119 \\
\hline ND, not determined. & & &
\end{tabular}




\section{Article}

respiratory epithelial cells should be used when analysing the antiviral activity of compounds that target host cell functions.

\section{Online content}

Any methods, additional references, Nature Research reporting summaries, source data, extended data, supplementary information, acknowledgements, peer review information; details of author contributions and competing interests; and statements of data and code availability are available at https://doi.org/10.1038/s41586-020-2575-3.

1. Wang, M. et al. Remdesivir and chloroquine effectively inhibit the recently emerged novel coronavirus (2019-nCoV) in vitro. Cell Res. 30, 269-271 (2020).

2. Ferner, R. E. \& Aronson, J. K. Chloroquine and hydroxychloroquine in covid-19. Br. Med. J. 369, $\mathrm{m} 1432$ (2020)

3. Touret, F. \& de Lamballerie, X. Of chloroquine and COVID-19. Antiviral Res. 177, 104762 (2020).

4. Hoffmann, M. et al. SARS-CoV-2 cell entry depends on ACE2 and TMPRSS2 and is blocked by a clinically proven protease inhibitor. Cell 181, 271-280 (2020).

5. Yao, X. et al. In vitro antiviral activity and projection of optimized dosing design of hydroxychloroquine for the treatment of severe acute respiratory syndrome coronavirus 2 (SARS-CoV-2). Clin. Infect. Dis. 71, 732-739 (2020).
6. Liu, J. et al. Hydroxychloroquine, a less toxic derivative of chloroquine, is effective in inhibiting SARS-CoV-2 infection in vitro. Cell Discov. 6, 16 (2020)

7. Rolain, J. M., Colson, P. \& Raoult, D. Recycling of chloroquine and its hydroxyl analogue to face bacterial, fungal and viral infections in the 21st century. Int. J. Antimicrob. Agents $\mathbf{3 0}$, 297-308 (2007).

8. Park, J. E. et al. Proteolytic processing of Middle East respiratory syndrome coronavirus spikes expands virus tropism. Proc. Natl Acad. Sci. USA 113, 12262-12267 (2016)

9. Shirato, K. Kawase, M. \& Matsuyama, S. Wild-type human coronaviruses prefe cell-surface TMPRSS2 to endosomal cathepsins for cell entry. Virology 517, 9-15 (2018).

10. Shirato, K., Kanou, K., Kawase, M. \& Matsuyama, S. Clinical isolates of human coronavirus 229E bypass the endosome for cell entry. J. Virol. 91, e01387-16 (2016).

11. Iwata-Yoshikawa, N. et al. TMPRSS2 contributes to virus spread and immunopathology in the airways of murine models after coronavirus infection. J. Virol. 93, e01815-18 (2019).

12. Zhou, Y. et al. Protease inhibitors targeting coronavirus and filovirus entry. Antiviral Res. 116, 76-84 (2015).

13. Matsuyama, S. et al. Enhanced isolation of SARS-CoV-2 by TMPRSS2-expressing cells. Proc. Natl Acad. Sci. USA 117, 7001-7003 (2020)

14. Boulware, D. R. et al. A randomized trial of hydroxychloroquine as postexposure prophylaxis for COVID-19. N. Engl. J. Med. 383, 517-525 (2020).

15. Kupferschmidt, K. Big studies dim hopes for hydroxychloroquine. Science 368, 1166-1167 (2020)

Publisher's note Springer Nature remains neutral with regard to jurisdictional claims in published maps and institutional affiliations.

(c) The Author(s), under exclusive licence to Springer Nature Limited 2020 


\section{Methods}

\section{Data reporting}

No statistical methods were used to predetermine sample size. The experiments were not randomized and the investigators were not blinded to allocation during experiments and outcome assessment.

\section{Cells}

Vero 76, Vero 76 stably expressing TMPRSS2 (both used for pseudotype experiments) ${ }^{4}$, the Vero 76 subclone Vero E6 (used for SARS-CoV-2 experiments), HEK293T and Calu-3 cells ${ }^{16}$ were cultured in Dulbecco's modified Eagle's medium (DMEM) or minimum essential medium (MEM, Calu-3) supplemented with $10 \%$ fetal bovine serum (FBS) and $1 \%$ penicillin-streptomycin. In case of Calu-3 cells, the medium was also supplemented with $1 \%$ non-essential amino acids and $1 \%$ sodium pyruvate. All cell lines were incubated at $37^{\circ} \mathrm{C}$ and $5 \% \mathrm{CO}_{2}$ and were obtained from repositories (Vero E6 and HEK293T) or collaborators (Calu-3 and Vero 76). Cell lines were free of mycoplasma, authenticated on the basis of morphology and growth properties and confirmed by PCR to be of the correct species. The cell lines used were not listed as commonly misidentified cell lines by the ICLAC register.

\section{Production of pseudotyped particles}

Vesicular stomatitis virus (VSV) particles pseudotyped with SARS-CoV-2 $\mathrm{S}$ were generated according to published protocols ${ }^{4,17}$. At $24 \mathrm{~h}$ after transfection, HEK293T cells expressing the S protein were inoculated with a replication-restricted, VSV-G-trans-complemented VSV, which lacks the genetic information for VSV-G but instead encodes the reporter genes $E G F P$ (enhanced green fluorescent protein) and FLuc (firefly luciferase), VSV* $\Delta \mathrm{G}$-FLuc ${ }^{18}$ (provided by G. Zimmer). After $1 \mathrm{~h}$ of incubation at $37^{\circ} \mathrm{C}$ and $5 \% \mathrm{CO}_{2}$, the inoculum was aspirated and the cells were washed with phosphate-buffered saline (PBS) before culture medium was added. The culture medium was further supplemented with the culture supernatant from I1-hybridoma cells (CRL-2700 cells, ATCC) containing anti-VSV-G antibody $(1: 1,000)$ to inactivate residual input virus. After an incubation period of $18 \mathrm{~h}$ at $37^{\circ} \mathrm{C}$ and $5 \% \mathrm{CO}_{2}$, the culture supernatant was collected, centrifuged to pellet cellular debris, and the clarified supernatant was aliquoted and stored at $-80^{\circ} \mathrm{C}$ until further use.

\section{Transduction of target cells with pseudotypes and its inhibition} For transduction experiments, Vero, TMPRSS2-expressing Vero and Calu-3 cells were grown in 96-well plates and allowed to reach about $50-70 \%$ confluency. Then, cells were preincubated with medium containing different concentrations $(10 \mathrm{nM}, 100 \mathrm{nM}, 1 \mu \mathrm{M}, 10 \mu \mathrm{M}$ or $100 \mu \mathrm{M}$ ) of camostat mesylate (Sigma-Aldrich), chloroquine or hydroxychloroquine (both Tocris) or DMSO (Roth, solvent control) for $2 \mathrm{~h}$ at $37^{\circ} \mathrm{C}$ and $5 \% \mathrm{CO}_{2}$, before they were inoculated with S-bearing VSV. At $18 \mathrm{~h}$ after transduction, culture supernatants were aspirated and cells were lysed by incubation ( $30 \mathrm{~min}$, room temperature) with Cell Culture Lysis Reagent (Promega). Cell lysates were subsequently transferred to white, opaque-walled 96-well plates and FLuc activity was quantified as an indicator of transduction efficiency, using the Beetle-Juice substrate (PJK) and a Hidex Sense plate reader (Hidex) operated with Hidex plate reader software (version 0.5.41.0, Hidex). Raw luminescence values (indicating luciferase activity) were recorded as counts per second. For normalization, transduction of DMSO-treated cells was set to $100 \%$ and the relative transduction efficiencies in the presence of camostat mesylate, chloroquine or hydroxychloroquine were calculated. Transduction experiments were performed in technical quadruplicates using three separate pseudotype preparations.

\section{SARS-CoV-2 infection of target cells and its inhibition}

Virus infections were done with SARS-CoV-2 Munich isolate 929. VeroE6 or Calu- 3 cells were seeded at densities of $3.5 \times 10^{5}$ cells per ml or $6 \times 10^{5}$ cells per $\mathrm{ml}$ in 12-well plates, respectively. After $24 \mathrm{~h}$, cells were incubated with chloroquine $(1 \mu \mathrm{M}, 10 \mu \mathrm{M}$ or $100 \mu \mathrm{M})$ or left untreated (control) for $1 \mathrm{~h}$ at $37^{\circ} \mathrm{C}$. Subsequently, cells were infected with an MOI of 0.001 in serum-free OPTIpro medium containing the above-mentioned chloroquine concentrations at $4{ }^{\circ} \mathrm{C}$ for $30 \mathrm{~min}$ to enable virus attachment. Afterwards, infection medium was removed and the wells were washed twice with PBS and DMEM supplemented with chloroquine was added as described above and the plates were incubated at $37^{\circ} \mathrm{C}$. Samples were taken at $24 \mathrm{~h}$ after infection. Infection experiments were conducted with biological triplicates in a biosafety level 3 laboratory.

\section{Viral RNA extraction and quantitative RT-PCR}

For viral RNA extraction from supernatants, $50 \mu \mathrm{l}$ of cell culture supernatant was mixed with RAV1 lysis buffer (Macherey-Nagel) followed by an incubation at $70^{\circ} \mathrm{C}$ for $10 \mathrm{~min}$. RNA extraction was performed as recommended by the manufacturer (Macherey-Nagel). For intracellular viral RNA extraction, cells were washed with PBS and lysed with TRIzol (Zymo). SARS-CoV-2 genome equivalents were detected by quantitative RT-PCR targeting the SARS-CoV-2 E gene as previously reported ${ }^{19}$, using the following primers: E_Sarbeco_F, ACAGGTACGTTAATAGTTAATAGCGT; E_Sarbeco_P1, FAM-ACACTAGCCATCCTTACTGCGCTTCG-BBQ; E_Sarbeco_R, ATATTGCAGCAGTACGCACACA. The quantitative RT-PCR experiment and data processing were carried out using the LightCycler 480 Real-Time PCR System (Roche) and LightCycler 480 Software (version 1.5, Roche Molecular Systems). Absolute quantification was performed using SARS-CoV-2-specific in vitro-transcribed RNA standards, as previously described ${ }^{19}$.

\section{Plaque assay}

Infectious SARS-CoV-2 plaque-forming units were quantified by plaque titration on Vero E6 cells, as previously described ${ }^{20}$, with minor modifications. Vero E6 monolayers were seeded in 24-well plates, washed with PBS, incubated with serial dilutions of SARS-CoV-2-containing cell culture supernatants in duplicates, and overlaid with $1.2 \%$ Avicel in DMEM, supplemented as described above. After $72 \mathrm{~h}$, cells were fixed with $6 \%$ formaline and visualized by crystal violet staining.

\section{Cell viability assay}

The cell viability was quantified using the CellTiter-Glo assay (Promega) and using the same experimental conditions as described above for transduction experiments with the exception that cells were not inoculated with virus particles. In brief, cells were preincubated for $2 \mathrm{~h}$ at $37^{\circ} \mathrm{C}$ and $5 \% \mathrm{CO}_{2}$ with medium containing different concentrations $(10 \mathrm{nM}$, $100 \mathrm{nM}, 1 \mu \mathrm{M}, 10 \mu \mathrm{M}$ or $100 \mu \mathrm{M}$ ) of camostat mesylate, chloroquine or hydroxychloroquine, or DMSO (solvent control), before culture medium was added (instead of medium containing VSV pseudotyped with SARS-CoV-2S) and cells were further incubated for $18 \mathrm{~h}$. Next, intracellular ATP levels were quantified as an indicator of cell viability. For this, culture supernatants were aspirated and cells were lysed by incubation with CellTiter-Glo substrate for $30 \mathrm{~min}$ at room temperature. Cell lysates were subsequently transferred to white, opaque-walled 96-well plates and luminescence was measured using a Hidex Sense plate reader (Hidex). Luminescence values (indicating cell viability) were recorded as absolute counts over a period of $200 \mathrm{~ms}$ per well. For normalization, cell viability of control-treated cells was set to $100 \%$ and the relative viability of cells incubated in the presence of camostat mesylate, chloroquine or hydroxychloroquine was calculated. Cell viability experiments were performed in technical quadruplicates and repeated with three separately prepared dilution series of the inhibitors.

\section{Statistical analysis}

Two-way ANOVA with Dunnett's post hoc test was performed to analyse statistical significance of differences in transduction efficiencies, SARS-CoV-2 genome equivalents or SARS-CoV-2 titres between control-and inhibitor-treated cells. $P>0.05$, not significant; ${ }^{*} P \leq 0.05$; 


\section{Article}

$\left.{ }^{* *} P \leq 0.01 ;{ }^{* * *} P \leq 0.001\right)$. The $\mathrm{IC}_{50}$ values, which indicate the inhibitor concentration that led to a $50 \%$ reduction in transduction, were calculated using a nonlinear regression model with variable slope. Statistical analyses and $\mathrm{IC}_{50}$ calculations were performed using GraphPad Prism (version 8.4.2).

\section{Reporting summary}

Further information on research design is available in the Nature Research Reporting Summary linked to this paper.

\section{Data availability}

All data are provided with the paper. Source data are provided with this paper.

16. Klemm, C. et al. Mitogen-activated protein kinases (MAPKs) regulate IL-6 over-production during concomitant influenza virus and Staphylococcus aureus infection. Sci. Rep. 7, 42473 (2017).

17. Kleine-Weber, $\mathrm{H}$. et al. Mutations in the spike protein of Middle East respiratory syndrome coronavirus transmitted in Korea increase resistance to antibody-mediated neutralization. J. Virol. 93, e01381-18 (2019).
18. Berger Rentsch, M. \& Zimmer, G. A vesicular stomatitis virus replicon-based bioassay for the rapid and sensitive determination of multi-species type I interferon. PLOS ONE $\mathbf{6}$ e25858 (2011).

19. Corman, V. M. et al. Detection of 2019 novel coronavirus (2019-nCoV) by real-time RT-PCR. Eurosurveill. 25, 2000045 (2020).

20. Herzog, P., Drosten, C. \& Müller, M. A. Plaque assay for human coronavirus NL63 using human colon carcinoma cells. Virol. J. 5, 138 (2008).

Acknowledgements This work was supported by the Bundesministerium für Bildung und Forschung (RAPID Consortium, 01KI1723A and 01KI1723D to C.D. and S.P., respectively). We thank A. Maisner (Vero 76 cell line), S. Ludwig (Calu-3 cell line) and G. Zimmer (VSV pseudotype system) for providing cell lines and reagents.

Author contributions M.H. and S.P. designed the study. M.H., K.M., H.H.-W., A.K., H.K.-W., N.K N.C.G. and M.A.M. performed research. M.H., M.A.M., C.D. and S.P. analysed the data. C.D. provided essential reagents. M.H. and S.P. wrote the manuscript. All authors revised the manuscript.

Competing interests The authors declare no competing interests.

\section{Additional information}

Supplementary information is available for this paper at https://doi.org/10.1038/s41586-020 2575-3.

Correspondence and requests for materials should be addressed to M.H. or S.P.

Reprints and permissions information is available at http://www.nature.com/reprints. 


\section{Reporting Summary}

Nature Research wishes to improve the reproducibility of the work that we publish. This form provides structure for consistency and transparency in reporting. For further information on Nature Research policies, see our Editorial Policies and the Editorial Policy Checklist.

\section{Statistics}

For all statistical analyses, confirm that the following items are present in the figure legend, table legend, main text, or Methods section.

n/a Confirmed

$\square$ The exact sample size $(n)$ for each experimental group/condition, given as a discrete number and unit of measurement

$\square$ \ A statement on whether measurements were taken from distinct samples or whether the same sample was measured repeatedly

The statistical test(s) used AND whether they are one- or two-sided

$\square$ Only common tests should be described solely by name; describe more complex techniques in the Methods section.

$\square$ \A description of all covariates tested

$\square$ \A description of any assumptions or corrections, such as tests of normality and adjustment for multiple comparisons

$\square$ A full description of the statistical parameters including central tendency (e.g. means) or other basic estimates (e.g. regression coefficient)

$\bigotimes$ AND variation (e.g. standard deviation) or associated estimates of uncertainty (e.g. confidence intervals)

$\square$ For null hypothesis testing, the test statistic (e.g. $F, t, r$ ) with confidence intervals, effect sizes, degrees of freedom and $P$ value noted

Give $P$ values as exact values whenever suitable.

Х $\square$ For Bayesian analysis, information on the choice of priors and Markov chain Monte Carlo settings

Х $\square$ For hierarchical and complex designs, identification of the appropriate level for tests and full reporting of outcomes

Х $\square$ Estimates of effect sizes (e.g. Cohen's $d$, Pearson's $r$ ), indicating how they were calculated

Our web collection on statistics for biologists contains articles on many of the points above.

\section{Software and code}

Policy information about availability of computer code

Data collection - Hidex Sense Microplate Reader Software (version 0.5.41.0), Hidex Deutschland Vertrieb GmbH, https://www.hidex.de/ - LightCycler ${ }^{\circledR} 480$ Software (version 1.5), Roche Molecular Systems, Inc., https://lifescience.roche.com/

Data analysis - GraphPad Prism (version 8.4.2), GraphPad Software, https://www.graphpad.com/

For manuscripts utilizing custom algorithms or software that are central to the research but not yet described in published literature, software must be made available to editors and reviewers. We strongly encourage code deposition in a community repository (e.g. GitHub). See the Nature Research guidelines for submitting code \& software for further information.

\section{Data}

Policy information about availability of data

All manuscripts must include a data availability statement. This statement should provide the following information, where applicable:

- Accession codes, unique identifiers, or web links for publicly available datasets

- A list of figures that have associated raw data

- A description of any restrictions on data availability 


\section{Field-specific reporting}

Please select the one below that is the best fit for your research. If you are not sure, read the appropriate sections before making your selection. \Life sciences

\section{Life sciences study design}

All studies must disclose on these points even when the disclosure is negative.

Sample size No sample size calculations were performed. Sample sizes adhere to standards in the field; for instance, three biological replicates performed with technical replicates.

Data exclusions No data were excluded from the analysis.

Replication All findings were confirmed in three independent experiments. All results could be reproduced.

Randomization Experiments were performed in vitro with standard immortalized cell lines. Therefore, no randomization was required.

Blinding No groups were allocated. Therefore, blinding was not necessary.

\section{Reporting for specific materials, systems and methods}

We require information from authors about some types of materials, experimental systems and methods used in many studies. Here, indicate whether each material, system or method listed is relevant to your study. If you are not sure if a list item applies to your research, read the appropriate section before selecting a response.

Materials \& experimental systems Methods

$\mathrm{n} / \mathrm{a}$ Involved in the study

\ $\square$ Antibodies

$\square \bigotimes$ Eukaryotic cell lines

\ $\square$ Palaeontology and archaeology $\mathrm{n} / \mathrm{a}$ Involved in the study

\ $\square$ Animals and other organisms

$\bigotimes \square$ Human research participants

\ $\square$ Clinical data

$\bigotimes \square$ Dual use research of concern

\ $\square$ ChIP-seq

\ $\square$ Flow cytometry

$\bigotimes \square$ MRI-based neuroimaging

\section{Eukaryotic cell lines}

Policy information about cell lines

Cell line source(s)

- Vero76: Provided by Andrea Maisner (Philipps University Marburg, Marburg/Germany)

- Vero76-TMPRSS2: Generated from parental Vero76 cells by retroviral transduction (Hoffmann et al., 2020)

- VeroE6: Obtained from ATCC (CCL-81)

- Calu-3: Provided by Stephan Ludwig (Institute of Virology (IVM), Westfaelische Wilhelms-University Muenster) and initially obtained from ATCC

-293T: Obtained from DSMZ (ACC 635)

Authentication

Mycoplasma contamination

Commonly misidentified lines (See ICLAC register)
Species origin of all cell lines was confirmed by sequencing a PCR-amplified fragment of the cytochrome c oxidase gene. Cell lines were further authenticated by analysis of morphology and growth properties.

Cells were tested for the presence of mycoplasma by PCR. No contamination by mycoplasma was detected.

The used cell lines are not listed as commonly misidentified cell lines by the ICLAC register. 